\title{
Dispositions d'un financement assuré de la sylviculture par le biais d'un système de tenure perpétuelle basé sur la location de la terre forestière
}

\section{Commentaire}

Cet article comprend les délibérations d'un atelier de travail de deux jours portant sur les mécanismes de financement de la sylviculture auquel ont participé des membres du comité exécutif national de l'ICF/CFI et du conseil de la division des bois et forêts de l'Association canadienne des pâtes et papiers. Il ne s'agit pas d'une position officielle. Cet article a été publié conjointement par les deux organisations pour promouvoir la discussion et la génération de nouvelles idées sur cette question importante.

Les commentaires des lecteurs sont des plus bienvenus et devraient être communiqués à C. Lee, directeur exécutif, $\mathrm{IFC} / \mathrm{CFI}$, à Ottawa.

\section{Préambule}

Il n'est pas question ici de tenter de concevoir un système national de tenure qui satisfasse à toutes les conditions et exigences à travers le pays. L'intention est plutôt de présenter des idées pour encourager l'évolution des systèmes de tenure au Canada.

L'orientation de ces idées porte essentiellement sur:

- Le développement durable

- Des mécanismes pour assurer le financement de la sylviculture

- Une nouvelle approche à la tenure des terres forestières publiques.

Au début d'octobre 1990, l'ACPP et l'Institut canadien des forêts/Canadian Institute of Forestry ont organisé un atelier de travail portant sur les mécanismes de financement de la sylviculture sur les terres forestières publiques.

Etant donné que l'investissement sylvicole par le secteur privé est étroitement lié à la structure et à la sécurité des ententes de tenue, l'intérêt a vite porté sur la tenure.

\section{Introduction}

Pendant la croissance de l'industrie forestière au Canada, du début des années 1800 jusqu'à maintenant, l'objectif principal des ententes ou licences de tenure forestière a porté sur un approvisionnement sûr de bois pour fins industrielles. Avec le temps, les licences ont règle générale prévu le reboisement après la récolte, mais ce n'était pas là l'objectif principal. Depuis 20 ans, les gouvernements provinciaux, le public et l'industrie repensent les priorités et cherchent à mettre au point des systèmes de tenure qui permettront le type d'aménagement forestier qu'exige la société moderne. Ce processus d'évolution des systèmes de tenure n'a pas réussi à répondre adéquatement aux besoins sociétaux qui évoluent rapidement.

Aux yeux de la plupart des observateurs, il est évident que le type d'aménagement forestier qu'il faudra dans les années
1990 comprendra une gestion éclairée de toutes les ressources forestières et cherchera à accroître l'envergure de tous les types d'avantages forestiers dont veux profiter la société. Ceux-ci sont économiques, environnementaux et d'utilisation non consommatrice, par ex. récréation, tourisme, etc.

Certains des objectifs spécifiques d'une telle intendance seront:

- le maintien de la qualité de l'eau et de l'habitat du poisson,

- le maintien de la biodiversité et de la gamme d'écosystèmes voulus pour perpétuer la variété naturelle de l'habitat faunique.

- le choix de pratiques forestières et de systèmes d'exploitation qui minimisera les effets enironnementaux.

- la diminution de la perturbation des sites,

- l'amélioration de l'esthétique de toutes les opérations,

- la régénération prompte après la récolte avec des essences propres au site qui contribueront au maintien des écosystèmes existants,

- l'augmentation de la croissance et du rendement.

La prochaine génération d'ententes de tenure forestière devra être ainsi conçue qu'elle mènera à un aménagement de la ressource forestière équitable tant pour la société que pour l'industrie forestière.

\section{Objectif du système de tenure}

Mettre au point un système de tenure qui permettra une bonne intendance de la ressource forestière sur les terres publiques et fournira à l'industrie ce dont elle a besoin pour tourner à profit dans une industrie en croissance.

L'un des buts d'un système révisé de tenure des terres forestières publiques est de voir au financement à long terme, stable et suffisant de la sylviculture. Si nous pouvons en arriver à de meilleurs sytèmes de tenure, les forêts aménagées du Canada croîtront mieux et donneront un meilleur rendement. En même temps, il y aura une augmentation des avantages tels que la récréation et la faune.

\section{Les intervenants et leurs intérêts}

L'aménagement des terres forestières publiques au Canada relève de quatre groupes principaux. Il s'agit du public, qui possède la terre et veut s'en servir pour fins récréatives, des gouvernements provinciaux, qui l'aménagent ou l'administrent au nom du public, des industries forestières, qui aménagent des terres forestières et récoltent le bois, et d'autres groupes qui se servent de la forêt pour fins commerciales non consommatrices (tourisme et pêche, pourvoyeurs de chasse et pêche, trappeurs et peuples autochtones). Chacun de ces groupes a des besoins et intérêts spécifiques. Un cinquième, le gouvernement fédéral, a un intérêt économique et environnemental général dans la gestion des terres forestières. 


\section{Le public}

Le public désire: que l'intendance de toutes les ressources des terres forestières publiques soit exemplaire, que les opérations de récolte aient des conséquences visuelles acceptables, que les opérations entravent le moins possible les autres usagers, que la ressource rende le maximum de revenus, que l'emploi soit sûr (principalement pour les gens des régions rurales) et qu'il y ait plus de facilités récréatives (principalement pour les gens de la ville). En somme, maximiser au fil des ans les retombées que désire la société.

\section{Gouvernements provinciaux}

Les gouvernements provinciaux désirent: que l'intendance de toutes les ressources forestières soit bonne, que l'industrie forestière soit saine et concurrentielle, que l'aménagement de la forêt favorise la croissance et la disponibilité du bois, mais avec un minimum de deniers publics, que la communauté soit prospère et stable, que les revenus soient le plus élevés possible (droits de coupe, impôts des particuliers et des compagnies), que les compagnies disposent de moins de droit d'aménagement des terres publiques, que les risques de l'aménagement soient partagés avec d'autres organismes, que la petite entreprise ait un maximum d'opportunité.

Ces petits groupes commerciaux ont des intérêts divers, y compris: tourisme, pourvoyeurs de chasse et pêche, guides et trappeurs, ainsi que les entrepreneurs en aménagement et opérations forestiers, tels que ceux qui s'occupent de la construction de routes, de la récolte, de la sylviculture, de l'inventaire forestier.

Les gouvernements voudraient aussi offrir plus de possibilités d'accès aux nouvelles entreprises s'intéressant à l'industrie du façonnage et aux autres activités centrées sur la forêt. Ceci intéresse tout particulièrement les gouvernements qui ne disposent que de peu ou pas de bois pour les nouveaux joueurs.

\section{L'industrie forestière}

L'industrie recherche: tenure certaine, approvisionnement stable ou croissant, d'accès à du bois en plus grandes quantités et de meilleure qualité, bois à prix concurrentiel et approbation de ses activités par le public. L'industrie estime que la demande mondiale pour des produits forestiers est à la hausse. Un aménagement forestier conçu pour augmenter le rendement que la société tire de toutes les ressources forestières enrichira la vie de tous les Canadiens. Sous l'emprise de directives appropriées, l'industrie a besoin de s'adonner à l'aménagement forestier et à l'expansion et à la diversification de ses installations de production avec un minimum de contrôle gouvernemental.

\section{Utilisation non consommatrice de la forêt}

Ceux dont les activités se fondent en tout ou en partie sur l'expérience de la nature sauvage ont besoin de milieux que l'activité industrielle ne perturbera pas, et tous ces groupes se fient beaucoup à une abondance de faune, de poisson et de sites naturels.

\section{Le gouvernement fédéral}

Les organismes fédéraux s'occupent d'une grande partie de la recherche forestière faite au Canada, et divers ministères ont des responsabilités touchant à la ressource forestière et à la faune, ainsi qu'une responsabilité générale concernant l'environnement.
Les suggestions de mécanismes de financement sylvicole et de tenure de terres forestières qu'on retiendra devront satisfaire à autant de ces besoins que possible.

\section{Proposition - Un système de tenure basé sur la location de terres forestières}

Les gouvernements et l'industrie devraient examiner un différent système de tenure des terres forestières qui favoriserait un financement sylvicole stable, ainsi qu'une intendance et un aménagement forestiers qui augmenteraient la croissance de la forêt.

(1) La tenure devrait être fondée sur le territoire, être reconduisable, et valable pour une période de $20-25$ ans. Les tenures seraient renouvelables à intervalles de cinq ans sur la base de la vérification du rendement.

(2) Le détenteur de la licence paierait des frais annuels de location de la capacité de production de bois du territoire et n'obtiendrait que des droits sur le bois, pas un droit exclusif à la terre, ni le droit de se servir des ou de consommer les autres valeurs.

Le loyer annuel serait calculé en fonction de facteurs tels que: site, sols, économique du transport pour avoir accès au terrain, essences, structure de la forêt par classes d'âge, possibilité annuelle sous aménagement de status quo, et coût de l'aménagement de toutes les valeurs couvertes par la licence. Il faudrait tenir compte d'une provision pour pertes normales dues aux feux, insectes et maladies. Possiblement aussi des provisions spéciales pour catastrophes.

Ce système de loyer annuel fixe, quel que soit le volume récolté, est inflexible. Des difficultés financières pourraient subvenir advenant perturbation grave de la production dans le sillage de récession, grèves, désastre en usine ou dans les juridictions où le gouvernement provincial aura légiféré sur les sources prioritaires d'approvisionnement en bois. $\mathrm{Ce}$ système ne réagit pas aussi bien au changement que le système traditionnel des droits de coupe.

(3) La possibilité annuelle du territoire sous licence au début de l'entente sera définie comme la productivité de ligne de base. Celle-ci sera le volume utilisé pour calculer le loyer annuel et sera fixée pour la période de l'entente (20-25 ans). L'aménagement du territoire sous bail devra voir à ce que la productivité de ligne de base soit maintenue. La productivité de ligne de base devra tenir compte des contraintes sur le volume récolté découlant de la distribution par classes d'âge, des facteurs environnementaux, etc. Le détenteur de la licence aura libre cours d'augmenter la productivité du territoire en choisissant les traitements sylvicoles qui offrent les meilleurs résultats. Les objectifs du plan d'aménagement seront son guide en tout temps.

(4) Le loyer du territoire sous bail devrait s'approcher du revenu net direct et justifiable que tire le gouvernement de ce territoire au moment de l'entrée en vigueur de l'entente. Le revenu net direct comprend les droits de coupe et de secteur, moins les frais de sylviculture, de mesurage, de protection de la forêt, etc. Il se pourrait qu'il faille faire des ajustements pour tenir compte des modifications apportées aux responsabilités quant à divers aspects de l'aménagement et aux frais réels de cet aménagement.

Le loyer devrait être rajusté annuellement en fonction du facteur déflationnaire du produit national brut pour tenir compte de l'impact de l'inflation. 
Ce loyer annuel sera payé quel que soit le volume de bois récolté (à moins de dispositions contraires). Le gouvernement en tirerait des revenus stables.

(5) Les ententes de tenure doivent être indépendantes de la Loi sur les terres publiques de la province et se fonder sur la Loi des contrats.

L'objectif est de mieux assurer la sécurité de la tenure mettant le contrat de tenure à l'abri d'une loi provinciale qui ne peut être modifiée au gré du gouvernement. Cependant, un mécanisme valable devra permettre de s'assurer que les objectifs de l'aménagement et la responsabilité qui en découle puissent être adaptés aux besoins de la société.

(6) Les ententes devront comprendre des dispositions d'arbitrage, advenant désaccord, et de compensation pour des pertes qui découleraient de modifications ou de défaut par l'une ou l'autre partie, ainsi que pour des retranchements de territoire.

(7) La licence devrait être une entente d'aménagement de la ressource forestière et son détenteur serait impliqué dans et aurait la responsabilité de l'aménagement et de l'intendance de toutes les ressources forestières. La responsabilité de l'aménagement des ressources autres que le bois serait déléguée par le biais de relations de travail pratiques avec les organismes gouvernementaux qui sont juridiquement responsables des sols, de l'habitat faunique, de l'habitat du poisson et d'autres telles ressources. Le détenteur intégrerait l'aménagement des ressources autres que le bois dans ses plans de gestion des opérations forestières. Ses droits ne porteraient que sur la capacité du territoire de produire du bois. L'aménagement en fonction de ces autres valeurs augmentera les frais du détenteur. Il faudra tenir compte de cet accroissement dans le calcul du loyer du territoire.

(8) Il faudra inclure dans les ententes des dispositions permettant au public de dire son mot quant aux objectifs d'aménagement et aux régimes d'exploitation. Une contribution primordiale devra provenir d'intéressés locaux légitimes. Des comités régionaux d'aménagement de la ressource forestière composés de représentants élus, de représentants de tous les groupes d'usagers de la ressource, de représentants communautaires et des peuples autochtones devraient déterminer le mixte des avantages à tirer du plan d'aménagement à long terme de chaque territoire sous licence. Ces gens devraient travailler en fonction des règlements provinciaux pour assurer la stabilité d'utilisation du territoire.

(9) L'industrie défraierait tous les traitements sylvicoles du territoire sous licence en plus du loyer annuel. Ceci pourra de faire de diverses façons:

- la sylviculture obligatoire de ligne de base reviendrait à l'industrie, soit directement soit par le biais d'un fonds en fidéicommis spécifique à la licence et détenu par une institution financière, ou garantie par une caution de bonne exécution, selon les circonstances.

- les traitements sylvicoles supplémentaires seraient à la charge du détenteur de la licence.

(10) La licence stipulerait que des études fiables de croissance et de rendement soient faites et que les augmentations de croissance qu'elles démontreraient soient acceptées comme telles, et que cette croissance supplémentaire, ainsi que tout effet de la possibilité réalisable soit à la disposition du détenteur de la licence pendant la durée de cette entente de 25 ans.

Les dispositions concernant les pratiques du détenteur en matière d'aménagement forestier, programmes sylvicoles compris, et de protection de la forêt devraient assurer la production des augmentations prévues de croissance. La possibilité annuelle sera accrue au fur et à mesure que les études de croissance et de rendement identifieront des augmentations de croissance. Il faudra prévoir des provisions pour toutes les petites exploitations actuelles qui tirent leur bois du territoire sous lincence.

(11) L'augmentation de la productivité de la forêt pendant la durée de l'entente (20-25 ans) n'entraînera pas d'augmentation du loyer.

(12) Le détenteur devra respecter les règlements normaux concernant la récolte annuelle et périodique de volumes et la possibilité annuelle. Le paiement du loyer au lieu des droits de coupe permettra de plus grandes variations qu'à l'ordinaire étant donné que le loyer sera fixe. L'impératif du "s'en servir ou la perdre" comme façon de prévenir la thésaurisation ne s'applique pas ici. Le paiement d'un loyer fixe pour une production de ligne de base donnée décourage cette thésaurisation.

(13) Des dispositions adéquates pourront être arrêtées concernant divers travaux à confier à de petites entreprises pour s'assurer d'un mixte socio-économique sain dans la région. L'aménagement intégré de la ressource forestière améliorera la disponibilité des autres ressources forestières au profit du public et des autres entrepreneurs.

\section{Avantages du système de tenure basé sur la location de la terre forestière}

\section{Avantages au public}

- le loyer des terres serait une injection stable de fonds dans les coffres publics;

- l'augmentation de la croissance et de la récolte se traduira par une activité économique accrue et de meilleurs revenus indirects;

- l'industrie défraie la sylviculture, ainsi que les frais d'aménagement et d'intendance des autres valeurs de la ressource;

- l'influence des intéressés légitimes sur la mise au point de la politique d'aménagement de la ressource et des priorités est assurée;

- l'aménagement intégré de la ressource forestière se réalisera et mènera à un meilleur éventail de bénéfices;

- le nombre et la diversité des emplois augmenteront;

- la prospérité et la stabilité de la communauté prendront du mieux grâce à l'augmentation de la disponibilité de bois et l'influence des intéressés locaux sur la politique;

- certaines recettes directes reviendraient aux communautés locales pour favoriser l'implication des intéressés légitimes.

\section{Avantages aux gouvernements}

- la plus grande disponibilité de fibre et la meilleure gamme d'autres bénéfices découlant de la ressource forestière avantageront la prospérité et la stabilité de la communauté;

- l'industrie defraie l'aménagement forestier et la sylviculture;

- l'augmentation des volumes de bois à prix concurrentiels rehaussera le niveau d'activité économique en général;

- les revenus directs de loyer (droits de coupe) sont stabilisés, les revenus indirects provenant de la taxation augmentent; 


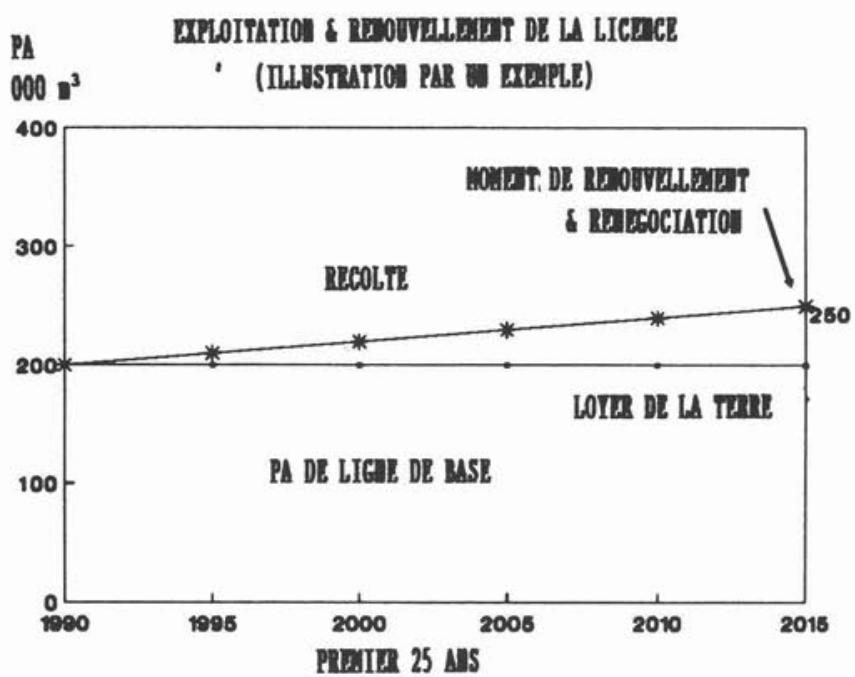

Figure 1. Exemple de fonctionnement de ce système.

- la petite entreprise sera stimulée, et les nouveaux entrepreneurs profiteront de nouvelles occasions d'affaires;

- l'apport du public et l'aménagement intégré de la ressource forestière diminueront l'impact négatif des opérations forestières. Ceci servira à améliorer le consentement/ approbation des opérations forestières par le public.

\section{Avantages à l'industrie}

Les effets de la sécurité de tenure et un système révisé de perception du loyer seront:

- l'encouragement à faire pousser plus de bois se traduira par une augmentation de la disponibilité;

- la sylviculture entraînera généralement une meilleure qualité du bois;

- le coût du bois sera plus prévisible;

- l'approbation du public devrait croître dans le sillage de la modification des objectifs d'aménagement.

Premier terme de 25 ans - un exemple

- La production de ligne de base est fixée à $200,000 \mathrm{~m}^{3} /$ année.

- Le loyer annuel est calculé en fonction de ce niveau de production, à la lumière d'autres facteurs importants: économiques, environnementaux et d'aménagement.
- A la fin de chaque période de cinq ans, l'aménagement serait évalué. Les régimes d'aménagement et d'exploitation et la sylviculture de ligne de base seraient soumis à vérification, et s'ils sont jugés satisfaisants la licence serait reconduite pour cinq ans de plus. Les objectifs d'aménagement pourraient également être examinés à ce moment-là.

- Pendant le terme de 25 ans, le détenteur de la licence s'occuperait de sylviculture de ligne de base et de sylviculture d'accroissement. Ces opérations pousseraient la production au niveau de $250000 \mathrm{~m}^{3} /$ an à la fin du terme. Les augmentations de croissance seraient identifiées et supportées par des études de croissance et de rendement.

Récolte possible pendant le terme de 25 ans:

- Ligne de base:

$$
25 \text { ans } \times 200,000 \mathrm{~m}^{3}=5,000,000 \mathrm{~m}^{3}
$$

- Augmentation:

$$
\begin{array}{lr}
25 \text { ans } \times 50,000 \mathrm{~m}^{3} \div 2= & 625,000 \mathrm{~m}^{3} \\
& 5,625,000 \mathrm{~m}^{3}
\end{array}
$$

L'augmentation de croissance de $625,000 \mathrm{~m}^{3}$ et sa récolte seraient libres de loyer, mais auraient été méritées par les frais sylvicoles encourus et la preuve de l'augmentation de production. Les gouvernements et le public profiteraient d'une hausse de l'activité économique et d'une augmentation des impôts des particuliers et des corporations.

\section{Définitions}

Production de ligne de base - La production du territoire sous licence basée sur l'accroissement annuel moyen des peuplements naturels actuels, ou comme le mentionnait le rapport de la Commission du Nouveau-Brunswick sur la mise en valeur de la forêt en 1957 " "...la récolte annuelle qui peut être faite sans aménagement scientifique de la forêt". Il ne faudrait pas interpréter ceci comme "un produit constant sans déclin", mais plus simplement la possibilité annuelle basée sur des peuplments naturels.

Sylviculture de ligne de base - Le niveau de sylviculture requis pour maintenir la production de ligne de base.

Sylviculture d'accroissement - Tout traitement sylvicole au-delà de la sylviculture de ligne de base. Tous ces traitements se traduiront par une augmentation de productivité. 